\title{
Autopercepción estética de la sonrisa en adultos Cuenca-Ecuador.
}

\section{Self - perception aesthetics of the smile in adult Cuenca-Ecuador.}

\author{
Vásquez, Cristina*1, Toral, Andrea ${ }^{1}$ y Reinoso, Napoleón ${ }^{1}$ \\ ${ }^{1}$ Universidad Católica de Cuenca \\ Cuenca, 010150, Ecuador \\ *avasquezp@ucacue.edu.ec
}

\begin{abstract}
Resumen
El objetivo de este estudio fue analizar la autopercepción de la estética de la sonrisa en pacientes que acudieron a la atención odontológica de la Universidad Católica de Cuenca. Fue un estudio descriptivo de corte transversal actual. Se aplicó una encuesta a 250 pacientes de 18 a 70 años de ambos sexos que asistieron a la consulta odontológica de la clínica docente de la Universidad Católica de Cuenca en el periodo Marzo - Julio del 2017, misma que consto de 11 preguntas relacionadas a la estética dental. Se obtuvo como resultado que la autopercepción de la estética dental fue mayor para el sexo femenino en un $12 \%$ más al comparar con el sexo masculino, en donde los factores de mayor afección fueron el color de dientes, posición en la que se encuentran, tamaño y forma en un 41,29\%,29,03\% y 22,58\% respectivamente, los de menor afección fueron diastemas y edentulismo; las piezas dentales más relacionadas a la autopercepción de la estética de la sonrisa fueron los incisivos centrales superiores e inferiores. Así podemos decir que todos los pacientes examinados auto percibieron al menos un factor que afectó la estética de la sonrisa.
\end{abstract}

Palabras clave: Estética, Autopercepción, Desdentamiento.

\begin{abstract}
The aim of this study was to analyze the self-perception of smile aesthetics in patients who attended dental care at the Catholic University of Cuenca. It was a descriptive study of current cross section, a survey was applied to 250 patients from 18 to 70 years of both sexes who attended the dental clinic of the teaching clinic of the Catholic University of Cuenca in the period March - July 2017, same which consists of 11 questions related to dental aesthetics. The result was that the self-perception of dental aesthetics was greater for the female sex by $12 \%$ more when compared to the male sex, where the factors of greatest affection were tooth color, position in which they are, size and formed in $41.29 \%, 29.03 \%$ and $22.58 \%$ respectively, those with the least affection were diastema and edentulism; The teeth most related to the self-perception of the aesthetics of the smile were the upper and lower central incisors. So, we can say that all the patients examined self-perceived at least one factor that affected their smile aesthetics.
\end{abstract}

Key words: Aesthetics, Self-perception, Edentulous.

\section{Introducción}

La autopercepción se define como un proceso en el cual los patrones de estímulos medio ambientales son organizados e interpretados, presentan una estrecha relación con el concepto de autoestima. El cual se puede conceptualizar como la manera en que una persona se mira a sí misma y establece conductas de rechazo o aprobación, autocalificándose por competencia y valor. El proceso de la autopercepción puede estar influenciado por un sinnúmero de factores psicológicos, culturales, físicos y sociales. ${ }^{1-3}$

La belleza facial con el paso del tiempo ha cambiado, la cara nos refleja los sentimientos y las emociones de una persona mientras que la sonrisa es la expresión de la vida ante la sociedad humana. ${ }^{4,5}$

Actualmente el atractivo facial es de gran importancia en las relaciones sociales, influye de manera directa con el éxito de la atracción del sexo opuesto, oportunidades, personalidad, rendimiento y perspectivas en el trabajo. En la interacción social, la boca es el medio de comunicación de la cara, por ende la sonrisa se considera primordial en la expresión facial y en la apariencia del individuo. ${ }^{6,7} \mathrm{El}$ paciente no solo busca una boca sana, sino también una sonrisa perfecta. ${ }^{8}$

La sonrisa es una parte esencial del estereotipo físico y la percepción del individuo, es la apreciación que las demás personas perciben de nuestra apariencia y personalidad, la armonía de la sonrisa juega un papel importante en la percepción de la belleza. ${ }^{9-11}$

El aspecto de la cavidad oral a veces es alterado por diversas enfermedades, es aquí donde el paciente empieza a mirar todos los rasgos que afectan su apariencia. ${ }^{8,12}$ En la actualidad todas las personas desean una sonrisa bonita, agradable, las personas encargadas de esto son los odontólogos, ofreciéndoles una serie de alternativas de un 
tratamiento exitoso. Es por esto que la necesidad dentro del campo de la odontología está relacionada con el concepto de estética que tiene el hombre en su mundo, el rostro es importante en la conjugación estética de un individuo y la sonrisa. $^{9}$

Los cambios generados por diversos motivos como el envejecimiento, edentulismo y alteraciones asociadas con problemas bucales afectan de manera significativa en la estética facial, afectando la percepción del paciente sobre sí mismo. De esta manera disminuye su autoestima, formando aislamiento y deterioro en su calidad de vida., 13-16

Los diversos factores que afectan la apariencia estética dental son el color, la forma, la posición de los dientes, la estética gingival, las restauraciones y el alineamiento dentario. Se considera que dentro de la satisfacción del paciente uno de los más importantes es el color de los dientes, posteriormente el correcto alineamiento8. El mayor causante de cambios faciales es el desdentamiento total o parcial. $^{1}$

En paciente con prótesis totales o parciales el odontólogo debe devolver al paciente la anatomía fisiológica del rostro, brindar una apariencia natural y una sonrisa armónica, reduciendo el daño psicológico causado por la pérdida de dientes, durante los tratamientos. ${ }^{17,18}$

Este estudio busca analizar la autopercepción de la estética de la sonrisa en pacientes que acudieron a la atención Odontológica de la Universidad Católica de Cuenca para de esta forma identificar la manera en cómo se siente y ve cada paciente con respecto a la estética de su sonrisa y la manera en que puede afectar en su comportamiento y en la seguridad de cada persona.

\section{Materiales y Métodos}

El presente es un estudio descriptivo, de corte transversal donde se aplicó una encuesta a 250 pacientes que asistieron a la consulta odontológica de la clínica docente de la Universidad Católica de Cuenca en el periodo Marzo - Julio del 2017, quienes cumplieron con los criterios de inclusión que fueron de estar en edad de 18 a 70 años, de ambos sexos, y exclusión no tener ninguna alteración estructural en el sistema.

Para la realización del estudio se utilizó una encuesta que constaba de once preguntas en la que se evaluaron varios parámetros de acuerdo a la sonrisa de cada paciente, y la autopercepción de la misma con el fin de respaldar la importancia dental y la importancia psicosocial de una sonrisa atractiva.

\section{Resultados}

Este estudio estuvo conformado por 250 pacientes distribuidos de acuerdo a sexo y edad con un porcentaje equitativo en relación al sexo, el $50 \%$ estuvo conformado por pacientes entre 18 y 28 años, el $24 \%$ de 29 - 39 años, el $13 \%$ de $40-50$ años, el $10 \%$ de 51 a 60 años y el $4 \%$ de 61 a 70 años. Véase tabla 1.
Tabla 1. Distribución de la población de acuerdo al sexo y la edad

\begin{tabular}{ccccccc}
\hline AD & \multicolumn{2}{c}{ FEMENINO } & \multicolumn{2}{c}{ MASCULINO } & \multicolumn{2}{c}{ TOTAL } \\
\hline $18-28$ & 64 & $26 \%$ & 60 & $24 \%$ & 124 & $50 \%$ \\
$29-39$ & 30 & $12 \%$ & 30 & $12 \%$ & 60 & $24 \%$ \\
$40-50$ & 15 & $6 \%$ & 17 & $7 \%$ & 32 & $13 \%$ \\
$51-60$ & 10 & $4 \%$ & 15 & $6 \%$ & 25 & $10 \%$ \\
$61-70$ & 2 & $1 \%$ & 7 & $3 \%$ & 9 & $4 \%$ \\
TOTAL & 121 & $48 \%$ & 129 & $52 \%$ & 250 & $100 \%$ \\
\hline
\end{tabular}

La autopercepción fue mayor en el sexo femenino que el masculino, $56 \%$ y $44 \%$ respectivamente. Véase gráfico 1. El factor de mayor afección fue color de los dientes con $42 \%$, seguido de posición dentaria con $29 \%$, tamaño y forma de las piezas con $23 \%$, fracturas $2 \%$ y para edentulismo, caries, apiñamiento y diastema $1 \%$ para cada caso. Véase gráfico 2.

Fig. 1. Autopercepción de la estética dental según el sexo en pacientes que asistieron a la clínica odontológica de la Universidad Católica de Cuenca

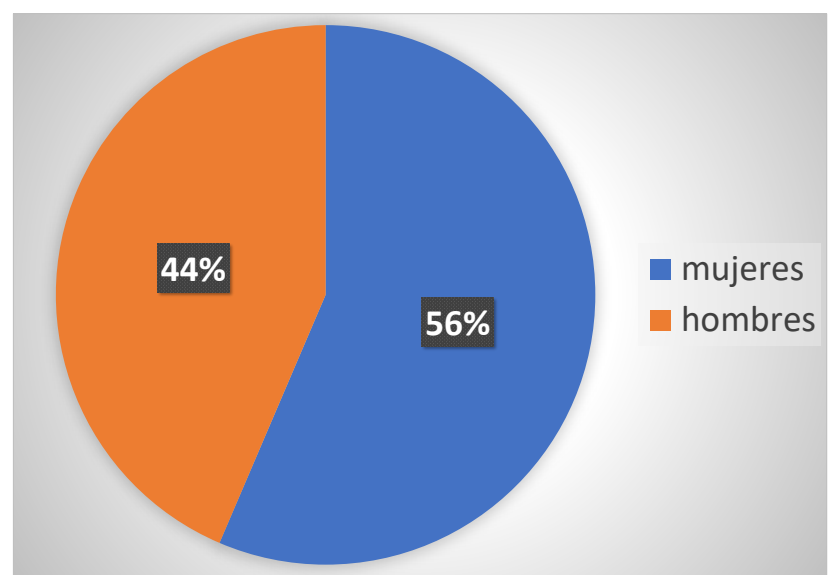

Fig. 2. Distribución de los factores de afección prevalentes en pacientes que asistieron a la clínica odontológica de la Universidad Católica de Cuenca

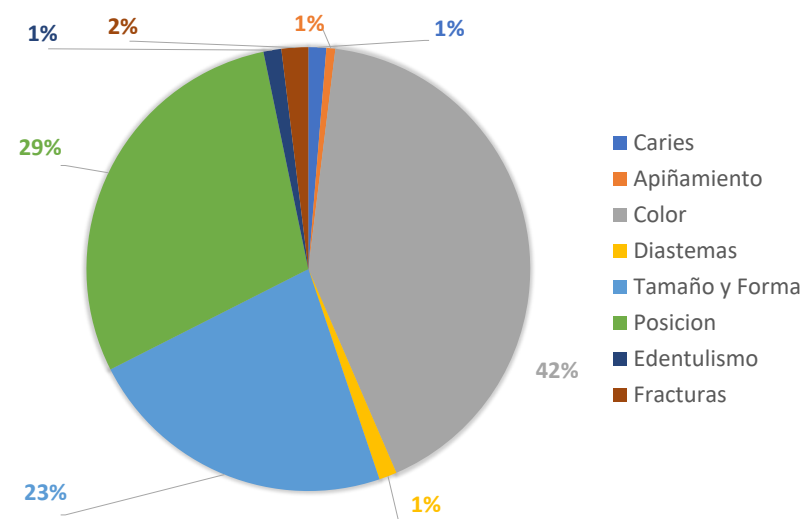

Las piezas más afectadas fueron los incisivos centrales superiores e inferiores, posteriormente los incisivos latera- 
les superiores e inferiores y caninos superiores e inferiores. Véase gráfico 3

Fig. 3. Distribución de las piezas más afectadas en pacientes que asistieron a la clínica odontológica de la Universidad Católica de Cuenca

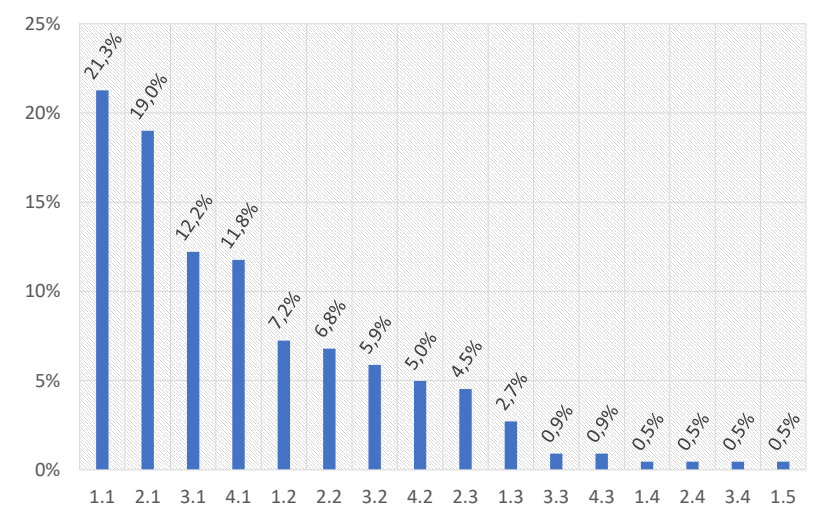

Fig. 4. Uso de prótesis según el sexo en pacientes que asistieron a la clínica odontológica de la Universidad Católica de Cuenca

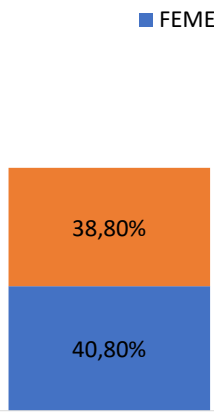

NO
MASCULINO
De los 250 pacientes el 20,40\% utilizó prótesis, en donde el $12,80 \%$ es de sexo masculino y el 7,60\% de sexo femenino. Véase gráfico 4 , en donde los pacientes entre los 40 a 50 años fueron los que más presentaban prótesis con un $6,40 \%$ y el rango de 18 a 28 años fue el menor con un $0,80 \%$, a comparación en los pacientes entre 61 y 70 años no presentan prótesis con un 48,80\%. Véase gráfico 5 .

El $22 \%$ de pacientes portadores de prótesis les hubiera gustado conservar sus dientes, el $16 \%$ y $15 \%$ les molesta el color y tamaño de los dientes de la prótesis respectivamente y al $6 \%$ les molesta el color de la encía. Véase gráfico 6.
Fig. 5. Distribución de pacientes que asistieron a la clínica odontológica de la Universidad Católica de Cuenca según el sexo y la edad

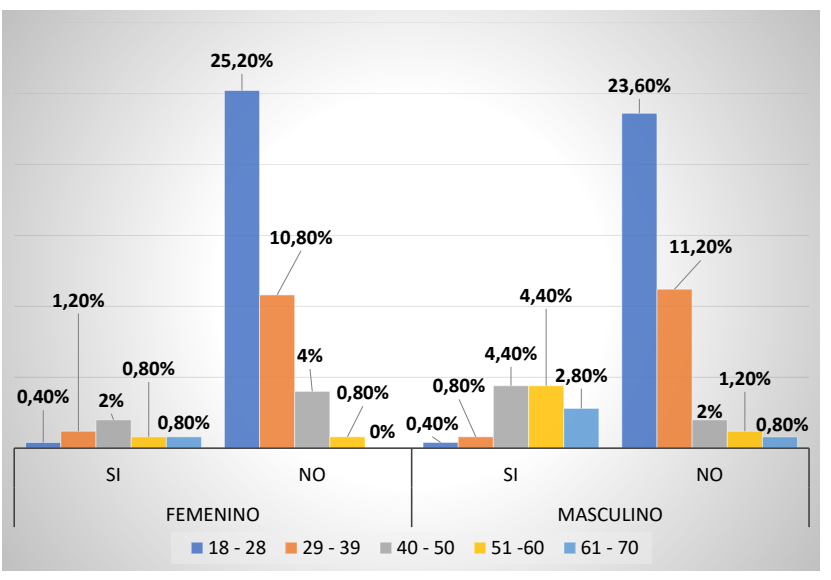

Fig. 6. Aspectos que a los pacientes que asistieron a la clínica odontológica de la Universidad Católica de Cuenca les gustaría modificar

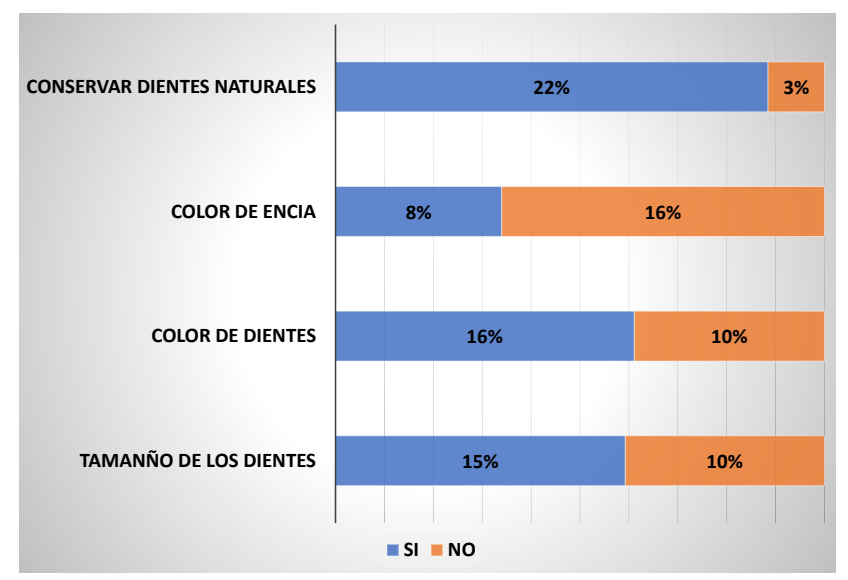

\section{Discusión}

La estética dental en los últimos años ha tomado gran relevancia en la clínica odontológica, ya que la mayoría de pacientes desean tener una óptima sonrisa y por ella han visto su interés en poseer una boca más limpia. La autopercepción de la persona dependerá de cómo se siente ella al mostrar su sonrisa, en varios casos existen pacientes que tienen diversos problemas bucales que conllevan a las personas a tener dificultades de baja autoestima y sus molestias van evolucionando. Es aquí donde el paciente opta por acudir al odontólogo y seguir un tratamiento óptimo para una correcta salud bucal. ${ }^{19}$

En este estudio las mujeres son las más afectadas en la autopercepción estética. Estudios similares como el de Márquez en el año 2014, menciona que también las mujeres se ven ampliamente afectadas al mostrar una autopercepción estética más negativa que los hombres, ya que el género femenino busca mejorías estéticas y cosméticas, lo mismo en su aspecto atractivo de los dientes y una 
sonrisa agradable. ${ }^{20}$ En un estudio realizado por Niesten et al. en el año 2012, los hombres son menos preocupados en comparación a las mujeres por su apariencia y que les importaba más la funcionalidad que la apariencia dental. ${ }^{21}$ Marchall en el 2012, menciona que las mujeres son más exigentes no solo a nivel oral, sino a nivel corporal, en la cual problemas relacionados con los mismos repercutirían a lo largo de toda su vida. ${ }^{22}$

Así también se obtuvo que el 20,4\% usa prótesis ya sea total o parcial, en el cual la mayoría de los pacientes les gustaría cambiar el aspecto de su prótesis, ya sea por el tamaño de los dientes, color de encía o de dientes. Fiske et al. han demostrado que pacientes portadores de prótesis han disminuido su confianza y una alteración en la socialización y en la forma de relacionarse ${ }^{23}$ además según Emani en el 2013, menciona que los pacientes portadores de prótesis presentan limitaciones funcionales. ${ }^{24}$

Pascual en el año 2006, manifiesta que de acuerdo a su investigación, el color de los dientes en una prótesis es un factor importante en la sonrisa. En su estudio da a conocer que el $65 \%$ de individuos se sienten bien con el color que usa y el $35 \%$ restante no les gusta cómo se observan sus piezas dentarias, es por ello que en comparación a este estudio en el cual el $20 \%$ no se sentían bien con el color de sus dientes, la mayoría de los pacientes se sentían conformes con el color de dientes de sus prótesis. ${ }^{25}$

Las piezas más afectadas en este estudio fueron los incisivos centrales superiores e inferiores con un $64,3 \%$. Por otro lado Alvino en el año 2009, comenta sobre la gran importancia que tienen las piezas anteriores, ya que son el centro de atención cuando un persona sonríe, en su estudio señala que el $72 \%$ de los pacientes se sienten insatisfechos con sus piezas anteriores y el $18 \%$ se siente contento con su sonrisa y dientes, ${ }^{4}$ Palma en el 2010 expone en su estudio que el $62,4 \%$ tiene una gran satisfacción sobre sus dientes anteriores y el 37,6\% quisieran mejorar la estética de su sonrisa, es por ello que él nos habla sobre una buena salud bucal que lleva el paciente.

Medina en el 2010 presenta la prevalencia que tienen las personas por problemas de baja autoestima y de cómo afecta a las personas con comentarios sobre su sonrisa, es por ello que muestra que el $32 \%$ se ve afectado por comentarios groseros y el $64 \%$ no presenta ningún problema emocional. ${ }^{26}$ Seguidamente Bersezio en el año 2016, manifiesta el gran porcentaje de personas afectadas por problemas con sus dientes, el $69 \%$ de las personas fueron afectadas por las burlas o maltratos sobre su sonrisa ya que la mayoría presentaba problemas periodontales y otros operatorios y el $31 \%$ se mostró con tranquilidad, ${ }^{25}$ en base a este estudio existían muchos factores que afectaban la percepción de la sonrisa como el color de los dientes con $42 \%$, seguido de posición dentaria con $29 \%$, tamaño y forma de las piezas con $23 \%$, fracturas $2 \%$ y para edentulismo, caries, apiñamiento y diastema $1 \%$.

Carey en el 2014 menciona que los pacientes desean tener dientes más blancos, no solamente a la presencia de manchas sino a la insatisfacción con el color natural de sus dientes. ${ }^{27}$ Kershaw en el 2008 ha correlacionado la presencia de dientes blancos con altos grados de competencia social, capacidad intelectual y relaciones interpersonales.28 Por otro lado Samorodnitsky en el 2007 menciona que problemas como la presencia de caries, restauraciones antiestéticas o antiguas y la ausencia de piezas dentales principalmente las anteriores conllevan a una insatisfacción de los pacientes frente a su apariencia. ${ }^{28}$

\section{Conclusión}

La autopercepción de la estética fue más afectada en el sexo femenino con un $56 \%$ que en el sexo masculino, con $44 \%$.

Los factores que presentaban mayor afección fue cambio de coloración de los dientes con un $42 \%$ y el de menor afección fue edentulismo, caries, apiñamiento, diastemas con $1 \%$.

Las piezas dentales más relacionadas a la autopercepción de la estética de la sonrisa fueron los incisivos centrales superiores e inferiores.

\section{Referencias Bibliográficas}

1. Orrego-Ramírez C, Meza-Fuentealba C, Vergara-Núñez C, Lee-Muñoz X, Schleyer-Daza N. Percepción estética del paciente desdentado comparada con la opinión de expertos: The aesthetic self-perception of the edentulous patient compared to the opinion of experts. Revista clínica de periodoncia, implantología y rehabilitación oral. 2016;9:54-58.

2. Lohigorry J, Tisera A, Lenta MM. SALUD MENTAL E INCLUSIÓN SOCIAL: DERECHO AL TRABAJO EN EL CONTEXTO ACTUAL. Facultad de Psicología; 2014. [Online; accessed 26. Dec. 2017]. Available from: https: //www.aacademica.org/maria.malena.lenta/75.

3. Rentería Guerrero VJ. Prevalencia de la proporción áurea en la sonrisa y factores asociados. Estudiantes de Odontología de la Universidad de Cuenca 2015 2016; 2016. Available from: http://dspace.ucuenca.edu. ec/handle/123456789/25781.

4. VALES MIA. ANÁLISIS DE LA SONRISA EN RELACIÓN A LAS PROPORCIONES FACIALES EN PACIENTES JÓVENES DE 17 A 20 AÑOS; 2012. [Online; accessed 26. Dec. 2017]. Available from: http://www.cop. org.pe/bib/tesis/MARIAISABELALVINOVALES.pdf.

5. López RPC. Mapeo facial de emociones sintéticas; 2005. [Online; accessed 26. Dec. 2017]. Available from: http: //tierra.aslab.upm.es/documents/PFC/PFC_RPConde.pdf.

6. Malquichagua Veramendi C, Liñán-Durán C, Ríos Villasis K. Percepción estética de la sonrisa con respecto a la inclinación de los incisivos superiores. Revista Estomatológica Herediana. 2014;24(3):155. Available from: http://www.upch.edu.pe/vrinve/dugic/revistas/ index.php/REH/article/view/2090.

7. Palmero Cantero F, e libro C. Manual de teorías emocionales y motivacionales. Castelló de la Plana: Universitat 
Jaume I, Servei de Comunicació i Publicacions; 2011. OCLC: 984775595.

8. Inda Álamos D. Evaluación de la autopercepción de la estética dental e impacto psicosocial en pacientes sometidos a blanqueamiento dental, seguimiento de 9 meses. Universidad de Chile; 2016. Available from: http: 20. //repositorio.uchile.cl/handle/2250/142444.

9. Mora MG, Serna MEV, Ledesma AF. Percepción de la estética de la sonrisa por odontólogos especialistas y pacientes; 2015. [Online; accessed 26. Dec. 2017]. Available from: http://www.medigraphic.com/pdfs/ortodoncia/ mo-2015/mo151c.pdf.

10. Zamora JAT. El conocimiento de sí mismo y la formación de la personalidad; 2005. [Online; accessed 26. Dec. 2017]. Available from: http://upnmorelos.edu.mx/assets/ cono_si_mismo_form_pers.pdf.

11. Kassin S, Fein S, Markus HR. Psicología social. México: 22 Wadsworth Cengage; 2010. OCLC: 870405031.

12. Gómez EJM. SALUD BUCODENTAL Y CALIDAD DE VIDA ORAL EN LOS MAYORES.; 2010. Available from: http://eprints.ucm.es/12303/1/T32521.pdf.

13. ORTIZ LMG. NIVEL DE SATISFACCIÓN DEL 23. Fiske J, Davis DM, Frances C, Gelbier S. The emotional USO DE PRÓTESIS TOTALES BIMAXILARES EN ADULTOS MAYORES DEL HOSPITAL DR. HUMBERTO MOLINA DE ZARUMA ENTRE 24. ABRIL DEL 2015 Y ENERO DEL AÑO 2016; 2016. [Online; accessed 26. Dec. 2017]. Available from: http://repositorio.ug.edu.ec/bitstream/redug/15793/ 1/GONZ\%c3\%81LEZ\%20ORTIZ.pdf.

14. Colom JM, Picand JB. Salud Oral y Calidad de Vida en el Adulto Mayor; 2001. [Online; accessed 26. Dec. 2017]. Available from: http://www.gerontologia.uchile.cl/ docs/biotti_2.htm.

15. Vidal SGV. Autopercepción-estética-funcionaldentomáxilo-facial-de adultos-desdentados-parciales.pdf; 26. 2013. [Online; accessed 26. Dec. 2017]. Available from: http://repositorio.uchile.cl/bitstream/handle/2250/ 130011/Autopercepci\%C3\%B3n-est\%C3\%A9ticafuncional-dentom $\%$ C $3 \%$ A 1 xilo-facial-de $\% 20$ adultosdesdentados-parciales.pdf? sequence $=1$.

16. Hernández RIE, Férez JJ, Rangel ALGC, Fulgencio AAG. Efecto de la depresión en la percepción de salud bucal y 27 . calidad de vida en adultos mayores - od062d.pdf; 2006. [Online; accessed 26. Dec. 2017]. Available from: http: //www.medigraphic.com/pdfs/adm/od-2006/od062d.pdf. 28

17. Lucas L, Gennari F, Goiato MC, Dos Santos D, Moreno A, Falcón-Antenucci R. Estética en prótesis removibles. Revista Cubana de Estomatología. 2010;47:224 - 235. Available from: http://scielo.sld.cu/scielo.php?script=sci_ arttext\&pid=S0034-75072010000200011\&nrm=iso.

18. Vélez BBS. I mportancia de la rehabilitación oral con prótesis removible flexible en pacientes con edentulismo parcial clase II división II en la clínica integral de la Facultad Piloto de Odontología periodo 2014 - 2015; 2015. [Online; accessed 26. Dec. 2017]. Available from: http://repositorio.ug.edu.ec/bitstream/redug/18149/
1/SUAREZbryan.pdf.

19. Espinosa DM, Pérez YJM. La Odontología estética como arte. Acta Médica del Centro. 2014;8(4):107-109. Available from: http://www.revactamedicacentro.sld.cu/index. php/amc/article/view/179.

0. Márquez Yáñez S. Autopercepción estética-funcional dento máxilo facial según grado de desdentamiento del sujeto adulto joven dentado completo, adulto dentado parcial y adulto mayor desdentado tota. Repositorio Académico - Universidad de Chile. 2014;Available from: http://repositorio.uchile.cl/handle/2250/130512.

21. Niesten D, van Mourik K, van der Sanden W. The impact of having natural teeth on the QoL of frail dentulous older people. A qualitative study. BMC Public Health. 2012 Dec;12(1). Available from: http://bmcpublichealth. biomedcentral.com/articles/10.1186/1471-2458-12-839.

. Marshall C, Lengyel C, Utioh A. Body Dissatisfaction: Among Middle-aged and Older Women. Canadian Journal of Dietetic Practice and Research. 2012 Jul;73(2):e241e241. Available from: http://dcjournal.ca/doi/10.3148/73. 2.2012.e241. effects of tooth loss in edentulous people. British Dental Journal. 1998 Jan;184(2):90-93; discussion 79.

4. Emami E, de Souza RF, Kabawat M, Feine JS. The Impact of Edentulism on Oral and General Health. International Journal of Dentistry. 2013;2013:1-7. Available from: http: //www.hindawi.com/journals/ijd/2013/498305/.

25. Pascual Moscardó A, Camps Alemany I. Odontología estética: Apreciacion cromática en la clínica y el laboratorio. Medicina Oral, Patología Oral y Cirugía Bucal. 2006 Jul;11(4):363-368. Available from: http://scielo.isciii.es/scielo.php?script=sci_ abstract\&pid=S1698-69462006000400015.

. Narváez SG, Ramírez CMM, Jaramillo PCB. PARÁMETROS ESTÁTICOS DE LA SONRISA ACEPTADOS POR ODONTÓLOGOS ESPECIALISTAS Y PACIENTES DE ORTODONCIA. Revista Colombiana de Investigación en Odontología. 2010 Sep;1(2). Available from: https://www.rcio.org/index.php/rcio/article/view/5/ 9\%3A\%3Ahtml.

7. Carey CM. Tooth Whitening: What We Now Know. journal of evidence-based dental practice. 2014 Jun;14 Suppl:70.

28. Samorodnitzky-Naveh GR, Geiger SB, Levin L. Patients' satisfaction with dental esthetics. J Am Dent Assoc. 2007 Jun;138(6):805-808. Available from: https://www.ncbi. nlm.nih.gov/pubmed/17545270.

Recibido: 24 de octubre de 2017

Aceptado: 19 de diciembre de 2017 
